\title{
Analytical normalization of resonant states in photonic crystal slabs and periodic arrays of nanoantennas at oblique incidence
}

\author{
T. Weiss, ${ }^{*}$ M. Schäferling, and H. Giessen \\ 4th Physics Institute and Research Center SCoPE, University of Stuttgart, Pfaffenwaldring 57, D-70550 Stuttgart, Germany \\ N. A. Gippius \\ Skolkovo Institute of Science and Technology, Novaya Street 100, Skolkovo 143025, Russia \\ S. G. Tikhodeev \\ Department of Physics, Lomonosov Moscow State University, Moscow 119991, Russia \\ and A. M. Prokhorov General Physics Institute, Russian Academy of Sciences, Vavilova Street 38, Moscow 119991, Russia
}

\author{
W. Langbein and E. A. Muljarov \\ Cardiff University, School of Physics and Astronomy, The Parade, Cardiff CF24 3AA, United Kingdom \\ (Received 21 March 2017; revised manuscript received 2 June 2017; published 20 July 2017)
}

\begin{abstract}
We present an analytical formulation for the normalization of resonant states at oblique incidence in one- and two-dimensional periodic structures with top and bottom boundaries to homogeneous space, such as photonic crystal slabs and arrays of nanoantennas. The normalization is validated by comparing the resonant state expansion using one and two resonant states with numerically exact results. The predicted changes of resonance frequency and linewidth due to perturbations of refractive index or geometry can be used to study resonantly enhanced refractive index sensing as well as the influence of disorder. In addition, the normalization is essential for the calculation of the Purcell factor.
\end{abstract}

DOI: 10.1103/PhysRevB.96.045129

\section{INTRODUCTION}

Micro- and nanophotonic structures have attracted significant interest in recent years. Their unique optical properties allow for realizing thin lenses [1,2], negative index materials $[3,4]$, perfect absorbers [5,6], and photonic band gaps $[7,8]$, to name a few.

The optical properties of micro- and nanophotonic structures are governed by their resonant states, also known as quasinormal or quasiguided modes. Resonant states in plasmonic nanoantennas and dielectric resonators modify the spontaneous emission rate of quantum emitters in their vicinity $[9,10]$, also known as the Purcell effect [11]. The control of the relative phase induced by resonant states in adjacent nanoantennas is the key element of metasurfaces [1,12]. Furthermore, resonant states can be used to enhance weak effects such as circular dichroism in chiral materials [13,14] and Faraday rotation in magneto-optical systems $[15,16]$. The reason is that small modifications in the dielectric surrounding can have a significant influence on the resonant states, which is also exploited in resonantly enhanced refractive index sensing [17,18].

Resonant states can be tailored by modification of size and shape of the involved constituents $[19,20]$, as well as by controlling the coupling of resonant states in different structures [21-25]. Planar periodic systems and other guiding geometries exhibit Rayleigh-Wood anomalies [26] that determine the optical properties of such systems in combination with the photonic band structure formed by the mutual coupling of the unit cells [27-32].

\footnotetext{
*t.weiss@pi4.uni-stuttgart.de
}

In general, resonant states are solutions of homogeneous Maxwell's equations, i.e., in the absence of sources or external fields, that fulfill outgoing boundary conditions and have discrete eigenfrequencies on the complex frequency plane. Thus, the resonant field distribution is only determined up to a scalar factor. Therefore, it is required to normalize the resonant states correctly in order to assign to each resonant state its appropriate weight in the optical response of the system.

The main difficulty in the normalization of the resonant states is encountered for resonant states that emit light to the far field, in contrast to bound or guided modes. The emission to the far field provides a radiative decay channel that is described by outward propagating waves due to causality. The corresponding imaginary part of the wave vector results in an amplitude of the resonant electromagnetic field that grows exponentially with the distance to the structure [see Fig. 1(b)]. The growing amplitude is reflecting the radiative decay: Light further away from the structure has left the system at an earlier time, at which more energy was stored in the resonator.

In order to normalize the resonant states, several approaches have been suggested [9,10,34-46]. This includes approximate formulations for high-quality modes [44-46], the utilization of perfectly matched layers [9] or, equivalently, complex coordinates [34] in the exterior, as well as numerical approaches $[42,43]$. A fully analytical form of normalization has been derived in Ref. [37] and applied to circular waveguides [38] as well as single particles [40]. In this approach, the normalization consists of a volume integral over an arbitrary volume $\mathcal{V}$ enclosing the structure of interest and a surface term on the surface $\partial \mathcal{V}$ of $\mathcal{V}$. The divergence of the fields in the volume contribution is compensated by the surface term. Recently, we have shown that this approach also results in the correct normalization for resonant states in periodic 

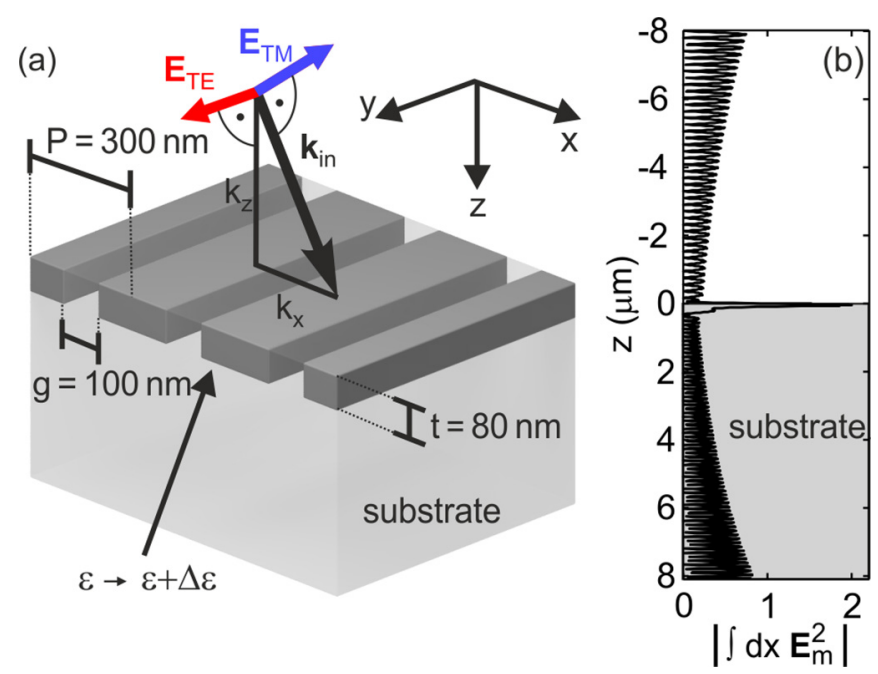

FIG. 1. (a) Schematic of the considered one-dimensional photonic crystal slab defining the relevant geometrical parameters. All parameters have been chosen following Ref. [33]. The periodic layer consists of materials with refractive indices of 1.5 and 2.5 , with the former being also the refractive index of the substrate. The superstrate is vacuum. (b) Photonic crystal slabs exhibit resonances that decay radiatively by emission to the far field. Consequently, the resonant field distribution grows exponentially to the exterior, as depicted here for the square of the electric field $\mathbf{E}_{m}$ of a transverseelectric (TE) mode at $k_{x}=5.236 \mu \mathrm{m}^{-1}$ and a resonance energy of $1891.4-12.6 i \mathrm{meV}$ integrated over one unit cell in the $x$ direction.

arrays of nanoantennas and photonic crystal slabs at normal incidence [42].

The analytical normalization has been used to develop the so-called resonant state expansion [37-41] for deriving the influence of both weak and strong modifications of a given system on its resonant states. Furthermore, it provides closed expressions for the Purcell factor in open resonators [10] as well as the sensitivity of resonant states in structures used for refractive index sensing [42].

In this article, we provide an analytical normalization for resonant states in periodic systems at oblique incidence. We validate it by comparing predictions based on the resonant state expansion with one and two modes with numerically exact results that are obtained using the Fourier modal method with adaptive coordinates [47], including the calculation of the resonant states $[42,48,49]$.

The resonant state expansion with a single mode determines the influence of refractive index changes on the resonance frequency and linewidth in a first-order perturbation theory [42,50], which allows for efficient optimization of refractive index sensing geometries. In the case of the two-mode resonant state expansion, we consider geometrical modifications, where the periodicity of the system is broken by introducing a perturbation in every second unit cell. Thus, we show that the resonant state expansion allows predicting the eigenfrequencies in systems with twice the period of the original system, while the computational domain is defined by the original unit cell. This approach can be easily extended to larger supercells in order to investigate the influence of disorder on the optical properties in periodic micro- and nanostructures
[51,52]. Furthermore, it has been shown that controlling the superperiodicity allows for tailoring the optical properties of photonic crystal slabs [53].

\section{EXAMPLE GEOMETRY}

Our analytical results are derived for two-dimensional periodic systems that are finite in the third direction. However, it is trivial to reformulate our theory to one- and threedimensional periodic systems. In the case of the latter, the normalization is trivial due to the absence of any surface terms, and only the volume contributions remain, with the volume of interest being the three-dimensional unit cell. In the case of the former, the system is periodic in one direction and translation invariant in the second, i.e., has an arbitrary periodicity in the second direction and is thus included in our treatment.

We consider henceforth a one-dimensional photonic crystal slab as example geometry [see Fig. 1(a)], consisting of a periodic layer $80 \mathrm{~nm}$ in thickness with period $P=300 \mathrm{~nm}$, in which $200 \mathrm{~nm} \mathrm{ZnO}(n=2.5)$ alternate with $100 \mathrm{~nm}$ quartz $(n=1.5)$. The substrate is quartz; the superstrate is air. Such systems exhibit transverse-electric (TE) and transversemagnetic (TM) quasiguided modes [28], which correspond to waveguide modes in the high-index material in the absence of periodicity. Due to the periodicity, the dispersion lines of the waveguide modes repeat periodically in reciprocal space with a period of $2 \pi / P$. The crossing of these dispersion lines is partially lifted, because the corresponding modes can couple depending on the symmetry of the electromagnetic fields and of the structure, forming so-called stop bands. Additionally, the periodicity allows coupling of the modes to the far field, resulting in a finite linewidth, and, thus, the quasiguided character of the modes.

We use Cartesian coordinates with $x$ along the direction of periodicity, $y$ along the direction of translation invariance, and $z$ along the finite extension of the structure, as shown in Fig. 1(a). Without the loss of generality, we choose $k_{y}=0$, so that the transverse-magnetic polarization corresponds to an electric field in the $x z$ plane, whereas transverse-electric polarization corresponds to an electric field along the $y$ direction.

\section{WAVE EQUATION IN PERIODIC SYSTEMS}

The starting point for our considerations is the wave equation, which follows from Maxwell's equations. For nonmagnetic systems $(\mu=1)$ with the spatially dependent permittivity tensor $\varepsilon(\mathbf{r} ; \omega)$ in the frequency domain [time dependence $\exp (-i \omega t)]$, the wave equation reads

$$
\mathcal{L}(\omega) \mathbf{E} \equiv-\nabla \times \nabla \times \mathbf{E}+\frac{\omega^{2}}{c^{2}} \varepsilon(\mathbf{r} ; \omega) \mathbf{E}=-i \frac{4 \pi \omega}{c^{2}} \mathbf{j},
$$

where $\mathcal{L}$ is defined as the corresponding operator acting on the electric field $\mathbf{E}$, and $\mathbf{j}$ is a current as the source of $\mathbf{E}$.

In the case of two-dimensional periodic structures, we can define the lattice vectors as

$$
\mathbf{R}=P_{1} n_{1} \hat{\mathbf{a}}_{1}+P_{2} n_{2} \hat{\mathbf{a}}_{2}\left(n_{1,2} \in \mathbb{Z}\right),
$$

such that $\varepsilon(\mathbf{r}+\mathbf{R} ; \omega)=\varepsilon(\mathbf{r} ; \omega)$. The directions of periodicity are in general not orthogonal and are specified by the unit 
vectors $\hat{\mathbf{a}}_{1}$ and $\hat{\mathbf{a}}_{2}$ with periods $P_{1}$ and $P_{2}$, respectively. The corresponding reciprocal lattice vectors are

$$
\mathbf{G}=\frac{2 \pi}{P_{1}} n_{1} \hat{\mathbf{b}}_{1}+\frac{2 \pi}{P_{2}} n_{2} \hat{\mathbf{b}}_{2} \quad\left(n_{1,2} \in \mathbb{Z}\right),
$$

with $\hat{\mathbf{a}}_{\alpha} \cdot \hat{\mathbf{b}}_{\beta}=\delta_{\alpha, \beta}\left(\delta_{\alpha, \beta}\right.$ denotes the Kronecker $\left.\delta\right)$.

Owing to the periodicity, we can apply Bloch's theorem, i.e., a solution of the wave equation (1) can be split into an exponential factor, $\exp (i \mathbf{k} \cdot \mathbf{r})$, and a periodic part. Here, $\mathbf{k}$ defines the in-plane momentum that is preserved apart from Umklapp processes throughout the whole structure. Henceforth, we consider a fixed $\mathbf{k}$ and its conjugate counterpart $-\mathbf{k}$ in our derivations. Note that in an experimental setup, $\mathbf{k}=0$ is associated with normal incidence, while $\mathbf{k} \neq 0$ corresponds to oblique incidence.

The top and bottom regions in the one- and two-dimensional periodic structures consist of homogenous and isotropic space. The permittivity values in the exterior are denoted by $\varepsilon^{\text {top }}$ and $\varepsilon^{\text {bot }}$, respectively. In addition, $\varepsilon^{\text {top }}$ and $\varepsilon^{\text {bot }}$ can be dispersive, but are assumed to be real.

A complete set of solutions in homogeneous space above and below the structure is given by the plane waves

$$
\mathbf{E}_{\mathbf{K}}^{p, \alpha}(\mathbf{r} ; \omega)=\psi_{\mathbf{K}}^{\alpha}(\mathbf{r} ; \omega) \hat{\mathbf{E}}_{\mathbf{K}}^{p, \alpha}(\omega),
$$

where

$$
\mathbf{K}=\mathbf{k}+\mathbf{G},
$$

$\alpha=\{$ top, bot $\}$, and $p=\{\mathrm{TE}, \mathrm{TM}\}$ denotes the linear polarization states. Furthermore,

$$
\psi_{\mathbf{K}}^{\alpha}(\mathbf{r} ; \omega)=\frac{1}{\sqrt{S_{\mathbf{u}}}} e^{i \mathbf{K} \cdot \mathbf{r}+i \kappa_{\mathbf{K}}^{\alpha}(\omega) \Delta z^{\alpha}}
$$

is a solution of the scalar wave equation in the top and bottom layers, respectively, with the area of one unit cell $S_{\mathrm{u}}$ and $\Delta z^{\alpha}=$ $\left|z-z^{\alpha}\right|$ specifying the distance to the $x y$ plane through the outermost interfaces between the periodic structure and the exterior at $z^{\alpha}$. The $z$ component of the corresponding wave vector is given by

$$
\kappa_{\mathbf{K}}^{\alpha}(\omega)= \pm \sqrt{\frac{\omega^{2}}{c^{2}} \varepsilon^{\alpha}(\omega)-|\mathbf{K}|^{2}} .
$$

Note that we define the sign in front of the square root to be positive for outgoing plane waves, while its negative counterpart denotes incoming plane waves. This requires that the branch cut for taking the complex square root has to be chosen such that Eq. (7) provides values on the positive (negative) real or imaginary axis for $\varepsilon^{\alpha} \omega^{2} / c^{2} \in \mathbb{R}$ and outgoing (incoming) boundary conditions. The normalized polarization vectors are

$$
\begin{gathered}
\hat{\mathbf{E}}_{\mathbf{K}}^{\mathrm{TE}, \alpha}=\hat{\mathbf{e}}_{z} \times \frac{i \mathbf{K}}{|\mathbf{K}|}, \\
\hat{\mathbf{E}}_{\mathbf{K}}^{\mathrm{TM}, \alpha}=\frac{-i}{\sqrt{\left(\kappa_{\mathbf{K}}^{\alpha}\right)^{2}+|\mathbf{K}|^{2}}}\left(s^{\alpha} \kappa_{\mathbf{K}}^{\alpha} \frac{\mathbf{K}}{|\mathbf{K}|}-|\mathbf{K}| \hat{\mathbf{e}}_{z}\right),
\end{gathered}
$$

with $\hat{\mathbf{E}}_{-\mathbf{K}}^{p, \alpha} \cdot \hat{\mathbf{E}}_{\mathbf{K}}^{p, \alpha}=1, s^{\text {top }}=-1$, and $s^{\text {bot }}=1$. The scalar waves obey the following orthonormality relation when integrating over one unit cell:

$$
\int_{\mathcal{S}_{\mathrm{u}}} d x d y \psi_{-\mathbf{K}}^{\alpha} \psi_{\mathbf{K}^{\prime}}^{\alpha}=\delta_{\mathbf{K}, \mathbf{K}^{\prime}} .
$$

This immediately results in an orthogonality relation for the vector waves in Eq. (4), given by

$$
\int_{\mathcal{S}_{u}} d x d y \mathbf{E}_{-\mathbf{K}}^{p, \alpha} \cdot \mathbf{E}_{\mathbf{K}^{\prime}}^{p^{\prime}, \alpha}=\delta_{\mathbf{K}, \mathbf{K}^{\prime}} \delta_{p, p^{\prime}}
$$

\section{A. Green's dyadic and resonant states}

The derivation of the solution of the wave equation (1) is in general nontrivial. Using the Green's dyadic $\mathcal{G}\left(\mathbf{r}, \mathbf{r}^{\prime} ; \mathbf{k} ; \omega\right)$ of a system, which is defined by the property

$$
\mathcal{L}(\omega) \mathcal{G}\left(\mathbf{r}, \mathbf{r}^{\prime} ; \mathbf{k} ; \omega\right)=\sum_{\mathbf{R}} \mathbb{1} \delta\left(\mathbf{r}-\mathbf{r}^{\prime}-\mathbf{R}\right),
$$

the electric field $\mathbf{E}$ generated by a source $\mathbf{j}$ can be calculated as

$$
\mathbf{E}(\mathbf{r} ; \mathbf{k} ; \omega)=-i \frac{4 \pi \omega}{c^{2}} \int_{\mathcal{V}} d V^{\prime} \mathcal{G}\left(\mathbf{r}, \mathbf{r}^{\prime} ; \mathbf{k} ; \omega\right) \mathbf{j}\left(\mathbf{r}^{\prime} ; \mathbf{k} ; \omega\right) .
$$

In the following, we extend the derivations in Ref. [42] to oblique incidence and show how we can express the Green's dyadic of one- and two-dimensional periodic systems in terms of the resonant states.

Resonant states are solutions of the wave equation (1) in the absence of sources (i.e., $\mathbf{j}=0$ ) with outgoing boundary conditions in the $z$ direction as defined according to Eqs. (6) and (7):

$$
\mathcal{L}\left[\omega_{m}(\mathbf{k})\right] \mathbf{E}_{m}(\mathbf{r} ; \mathbf{k})=0 .
$$

The eigenfrequencies $\omega_{m}(\mathbf{k})$ are in general complex with the real part denoting the resonance frequency and $-2 \operatorname{Im}\left(\omega_{m}\right)$ being the resonance linewidth. Owing to reciprocity, $\omega_{m}(\mathbf{k})=$ $\omega_{m}(-\mathbf{k})$. Furthermore, for every resonant state at the eigenfrequency $\omega_{m}$, there exists a resonant state with the electric field distribution $\mathbf{E}_{m}^{*}$ at the eigenfrequency $-\omega_{m}^{*}$.

Let us introduce the field $\tilde{\mathbf{E}}_{m}(\mathbf{r} ; \mathbf{k} ; \omega)$ as a solution of the differential equation

$$
\mathcal{L}(\omega) \tilde{\mathbf{E}}_{m}(\mathbf{r} ; \mathbf{k} ; \omega)=\frac{\omega^{2}-\omega_{m}^{2}}{c^{2}} \sigma_{m}(\mathbf{r} ; \mathbf{k}) .
$$

The vector of the periodic source $\sigma_{m}$ is proportional to the current $\mathbf{j}$ localized inside the periodic structure, i.e., is zero above and below it. In the limit $\omega \rightarrow \omega_{m}$, this results in the eigenvalue equation (14) for the resonant state $\mathbf{E}_{m}$. In combination with Eq. (13), we obtain

$$
\begin{aligned}
\mathbf{E}_{m}(\mathbf{r} ; \mathbf{k}) & =\lim _{\omega \rightarrow \omega_{m}} \tilde{\mathbf{E}}(\mathbf{r} ; \mathbf{k} ; \omega) \\
& =\lim _{\omega \rightarrow \omega_{m}} \int_{\mathcal{V}} d V^{\prime} \mathcal{G}\left(\mathbf{r}, \mathbf{r}^{\prime} ; \mathbf{k} ; \omega\right) \frac{\omega^{2}-\omega_{m}^{2}}{c^{2}} \sigma_{m}(\mathbf{r} ; \mathbf{k}) .
\end{aligned}
$$

Note that the source term in Eq. (15) has a rather simple form. In principle, the main requirement is that the right-hand side of Eq. (15) vanishes in the limit $\omega \rightarrow \omega_{m}$ with the frequency behavior $\propto\left(\omega-\omega_{m}\right)$. Hence, we assume that the eigenfrequencies $\omega_{m}$ must be simple poles of $\mathcal{G}$. In addition, the behavior of $\mathcal{G}$ on the complex frequency plane is determined by cuts arising from the two branches of $\kappa_{\mathbf{K}}^{\alpha}$ in Eq. (7), 
one pair for each reciprocal lattice vector $\mathbf{G}$. For nonabsorbing media in the exterior half spaces, these cuts start at branch points on the real frequency axis at the Rayleigh anomalies $\omega_{\mathbf{K}}$. Furthermore, the cuts must not cross the real frequency axis. We choose them to extend to the half plane with a negative imaginary part, which is suited also for absorbing media. At the Rayleigh anomalies, certain solutions of the homogeneous wave equation in the exterior change their character from evanescent to propagating in the $z$ direction. This opening or closing of far-field channels occurs for $\kappa_{\mathbf{K}}^{\alpha}=0$, and it influences the spectral line shape [26].

In summary, the behavior of $\mathcal{G}$ is determined by a countable number of poles and cuts on the complex frequency plane, so that we can use the Mittag-Leffler theorem [39,54,55] and write

$$
\mathcal{G}\left(\mathbf{r}, \mathbf{r}^{\prime} ; \mathbf{k} ; \omega\right)=\sum_{m} \frac{\mathcal{R}_{m}\left(\mathbf{r}, \mathbf{r}^{\prime} ; \mathbf{k}\right)}{\omega-\omega_{m}}+\sum_{\omega_{\mathbf{K}}} \mathcal{G}_{\omega_{\mathbf{K}}}\left(\mathbf{r}, \mathbf{r}^{\prime} ; \mathbf{k} ; \omega\right) .
$$

The first term on the right-hand side arises from the poles with residues $\mathcal{R}_{m}$, the second term denotes the cut contributions $[38,39,55]$ :

$$
\mathcal{G}_{\omega_{\mathbf{K}}}\left(\mathbf{r}, \mathbf{r}^{\prime} ; \mathbf{k} ; \omega\right)=\frac{1}{2 \pi i} \int_{\omega_{\mathbf{K}}-i \infty}^{\omega_{\mathbf{K}}} d \omega^{\prime} \frac{\Delta \mathcal{G}_{\omega_{\mathbf{K}}}\left(\mathbf{r}, \mathbf{r}^{\prime} ; \mathbf{k} ; \omega^{\prime}\right)}{\omega-\omega^{\prime}} .
$$

The integrand contains the difference in the Green's dyadic when approaching the cut from opposite sides; i.e., for $\omega$ on the cut, we have

$$
\Delta \mathcal{G}_{\omega_{\mathbf{K}}}\left(\mathbf{r}, \mathbf{r}^{\prime} ; \mathbf{k} ; \omega\right)=\lim _{\epsilon \rightarrow 0} \mathcal{G}\left(\mathbf{r}, \mathbf{r}^{\prime} ; \mathbf{k} ; \omega+\epsilon\right)-\mathcal{G}\left(\mathbf{r}, \mathbf{r}^{\prime} ; \mathbf{k} ; \omega-\epsilon\right) .
$$

Note that the cut is not defined uniquely on the complex frequency plane [55], and Eq. (18) just provides an example of cut contributions.

If we assume the normalization condition

$$
\int_{\mathcal{V}} d V \mathbf{E}_{m}(\mathbf{r} ;-\mathbf{k}) \cdot \sigma_{m}(\mathbf{r} ; \mathbf{k})=1+\delta_{\omega_{m}, 0},
$$

the application of Eq. (17) in Eq. (16) results due to reciprocity (see Appendix C) in

$$
\begin{aligned}
\mathcal{G}\left(\mathbf{r}, \mathbf{r}^{\prime} ; \mathbf{k} ; \omega\right)= & c^{2} \sum_{m} \frac{\mathbf{E}_{m}(\mathbf{r} ; \mathbf{k}) \otimes \mathbf{E}_{m}\left(\mathbf{r}^{\prime} ;-\mathbf{k}\right)}{2 \omega_{m}(\mathbf{k})\left[\omega-\omega_{m}(\mathbf{k})\right]} \\
& +\sum_{\omega_{\mathbf{K}}} \mathcal{G}_{\omega_{\mathbf{K}}}\left(\mathbf{r}, \mathbf{r}^{\prime} ; \mathbf{k} ; \omega\right),
\end{aligned}
$$

where $\otimes$ denotes the outer vector product. It is worth mentioning that Eq. (21) is only valid up to the outermost interfaces in $z$ direction; i.e., it cannot be used in the homogeneous top and bottom half spaces above and below the spatially inhomogeneous structure of interest. From a physical point of view, this is clear, because the Green's dyadic must not grow exponentially in the exterior. Mathematically, it is a direct consequence of the applicability of the Mittag-Leffler theorem, which requires for Eq. (21) that the Green's dyadic remains finite and analytical in the whole complex frequency plane except for a countable number of poles and the cuts [54]. This condition, however, cannot be fulfilled in the exterior regions due to the outgoing boundary conditions.
The condition in Eq. (20) is similar to that provided in Ref. [40], but it contains the conjugated resonant electric field at $-\mathbf{k}$. In the case of degenerate resonant states, the source terms $\sigma_{m}$ have to be chosen such that they ensure that $\int_{V} d V \mathbf{E}_{m}(\mathbf{r} ;-\mathbf{k}) \cdot \sigma_{m^{\prime}}(\mathbf{r} ; \mathbf{k})=\delta_{m, m^{\prime}}$.

One can formulate the cut contributions in a way similar to that of the poles:

$$
\begin{aligned}
& \mathcal{G}_{\omega_{\mathbf{K}}}\left(\mathbf{r}, \mathbf{r}^{\prime} ; \mathbf{k} ; \omega\right) \\
& \quad=c^{2} \int_{\omega_{\mathbf{K}}-i \infty}^{\omega_{\mathbf{K}}} d \omega^{\prime} \frac{\mathbf{E}_{\omega_{\mathbf{K}}}\left(\mathbf{r} ; \mathbf{k} ; \omega^{\prime}\right) \otimes \mathbf{E}_{\omega_{\mathbf{K}}}\left(\mathbf{r}^{\prime} ;-\mathbf{k} ; \omega^{\prime}\right)}{2 \omega^{\prime}\left(\omega-\omega^{\prime}\right)} .
\end{aligned}
$$

Here, the fields $\mathbf{E}_{\omega_{\mathbf{K}}}$ are eigensolutions of Eq. (14) without the requirement of outgoing boundary conditions.

\section{B. Normalization}

Equation (21) contains electric field distributions $\mathbf{E}_{m}$ as solutions of Eq. (14). However, in Eq. (14), the fields $\mathbf{E}_{m}$ are only fixed up to an arbitrary scalar factor. Therefore, one has to determine the correct factor for $\mathbf{E}_{m}$ in Eq. (21), i.e., the correct normalization of the resonant states. Similarly to the derivations in Ref. [10] and references therein, this can be achieved by substituting $\sigma_{m}$ from Eq. (15) into Eq. (20) and subtracting a zero in the form of an integrand based on Eq. (14), which yields

$$
\begin{aligned}
1 & +\delta_{\omega_{m}, 0}=\lim _{\omega \rightarrow \omega_{m}} \frac{c^{2}}{\omega^{2}-\omega_{m}^{2}} \\
& \times \int_{\mathcal{V}} d V\left[\mathbf{E}_{m}^{-} \cdot \mathcal{L}(\omega) \tilde{\mathbf{E}}_{m}^{+}(\omega)-\tilde{\mathbf{E}}_{m}^{+}(\omega) \cdot \mathcal{L}\left(\omega_{m}\right) \mathbf{E}_{m}^{-}\right] .
\end{aligned}
$$

For brevity of notations, we introduce here

$$
\mathbf{E}_{m}^{ \pm} \equiv \mathbf{E}_{m}( \pm \mathbf{k}), \quad \tilde{\mathbf{E}}_{m}^{ \pm}(\omega) \equiv \tilde{\mathbf{E}}_{m}( \pm \mathbf{k} ; \omega) .
$$

After some algebra (see Appendix B), one obtains the normalization condition

$$
1+\delta_{\omega_{m}, 0}=I_{m}^{\mathcal{V}}+\left.\frac{\partial S_{m}^{\partial \mathcal{V}}}{\partial\left(\omega^{2}\right)}\right|_{\omega_{m}},
$$

with the volume term

$$
\left.I_{m}^{\mathcal{V}} \equiv \int_{\mathcal{V}} d V \mathbf{E}_{m}^{-} \cdot \frac{\partial\left(\omega^{2} \varepsilon\right)}{\partial\left(\omega^{2}\right)}\right|_{\omega_{m}} \mathbf{E}_{m}^{+}
$$

and the surface term

$$
S_{m}^{\partial \mathcal{V}}(\omega) \equiv c^{2} \oint_{\partial \mathcal{V}} d S\left[\mathbf{E}_{m}^{-} \cdot \partial_{s} \tilde{\mathbf{E}}_{m}^{+}(\omega)-\tilde{\mathbf{E}}_{m}^{+}(\omega) \cdot \partial_{s} \mathbf{E}_{m}^{-}\right]
$$

Here, $\partial_{s}$ denotes the derivative in the direction of the surface normal.

The field distribution $\tilde{\mathbf{E}}_{m}$ depends on the source $\sigma_{m}$ in Eq. (15), so that it seems to be difficult to evaluate Eq. (27) and its derivative with respect to $\omega^{2}$ at $\omega_{m}$. However, due to the periodicity of the fields, the surface contributions at the periodic boundaries vanish, and only the contributions at the surfaces in the homogeneous top and bottom regions remain.

The fields in the exterior (indicated by $\mathbf{r}_{>}^{\alpha}$ with $\alpha=$ \{top, bot\}) can be decomposed into plane waves:

$$
\tilde{\mathbf{E}}_{m}\left(\mathbf{r}_{>}^{\alpha} ; \mathbf{k} ; \omega\right)=\sum_{\mathbf{K}, p} \tilde{a}_{\mathbf{K}}^{p, \alpha}(\omega) \mathbf{E}_{\mathbf{K}}^{p, \alpha}\left(\mathbf{r}_{>}^{\alpha} ; \omega\right) .
$$


Note that we omitted the mode index $m$ in the expansion coefficient $\tilde{a}_{\mathbf{K}}^{p, \alpha}$ for the sake of brevity. The exact form of the source $\sigma_{m}$ influences the frequency dependence of $\tilde{a}_{\mathbf{K}}^{p, \alpha}$, while

$$
a_{\mathbf{K}}^{p, \alpha} \equiv \tilde{a}_{\mathbf{K}}^{p, \alpha}\left(\omega_{m}\right)
$$

is solely determined by the resonant field distribution. As shown in Appendix B, it is sufficient to know $a_{\mathbf{K}}^{p, \alpha}$ for the evaluation of Eq. (27), because the derivative of $S_{m}^{\partial \mathcal{V}}$ with respect to $\omega^{2}$ only depends on $\tilde{a}_{\mathbf{K}}^{p, \alpha}\left(\omega_{m}\right)$.

The resulting surface contribution in Eq. (25) can in principle be evaluated for arbitrary surfaces in the exterior (see Appendix B). The simplest expressions are found when assuming that the surfaces are planes normal to the $z$ direction. In this case, the correct analytical normalization can be written as

$$
1+\delta_{\omega_{m}, 0}=I_{m}^{\mathcal{V}}+S_{m}^{\text {top }}+S_{m}^{\text {bot }}
$$

with the top and bottom surfaces being located at relative positions $\Delta z^{\alpha}$ in the exterior:

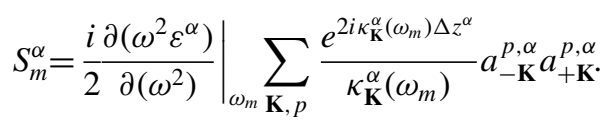

Note that the surface contributions vanish for guided modes with $\operatorname{Im}\left(\kappa_{\mathbf{K}}^{\alpha}\right)>0 \forall \mathbf{K}$ in the limit $\Delta z^{\alpha} \rightarrow \infty$, providing the expected result that the normalization of guided modes can be obtained by integrating the volume term in the $z$ direction from $-\infty$ to $\infty$. Furthermore, Eq. (31) results in Eq. (5) of Ref. [42] when considering normal incidence and $\Delta z^{\alpha}=0$, but at oblique incidence, it requires knowing also the conjugate form of the resonant electric field.

In the case of resonances that emit light to the far field, the exponentially growing contribution in the volume term is exactly compensated by the surface term. Intuitively, the volume and surface integrals are related to the energy stored in the volume and the energy flux through the surface. The part of the volume term corresponding to emission, which should not be used for the normalization, increases exponentially with distance and is removed by the surface term. Figure 2 illustrates this behavior for the transverse-electric resonance of the example structure at $k_{x}=\pi / 2 P \approx 5.236 \mu \mathrm{m}^{-1}$ and a resonance energy of $1891.4-12.6 i \mathrm{meV}$. As indicated in Fig. 2(a), we extend the volume of integration to the substrate region starting from the minimum volume $\mathcal{V}_{0}$ containing the outermost interface of the structure, i.e., the minimum volume containing all the inhomogeneities of the permittivity within the unit cell. The resonant electric field distribution depicted in Fig. 2(b) has been normalized using Eq. (30) based on this minimum volume. In Fig. 2(c), we show how the additional volume contribution in the substrate diverges for increasing volume of integration using the normalized fields (black solid line, left axis). The red solid curve demonstrates the cancellation of the additional volume contribution by the modification of the surface term in Eq. (30) based on our numerical calculations. The cancellation within the range of $10^{-12}$ shows the validity of the normalization, with the nonzero value being due to limitations of the accuracy of our numerical scheme [47].
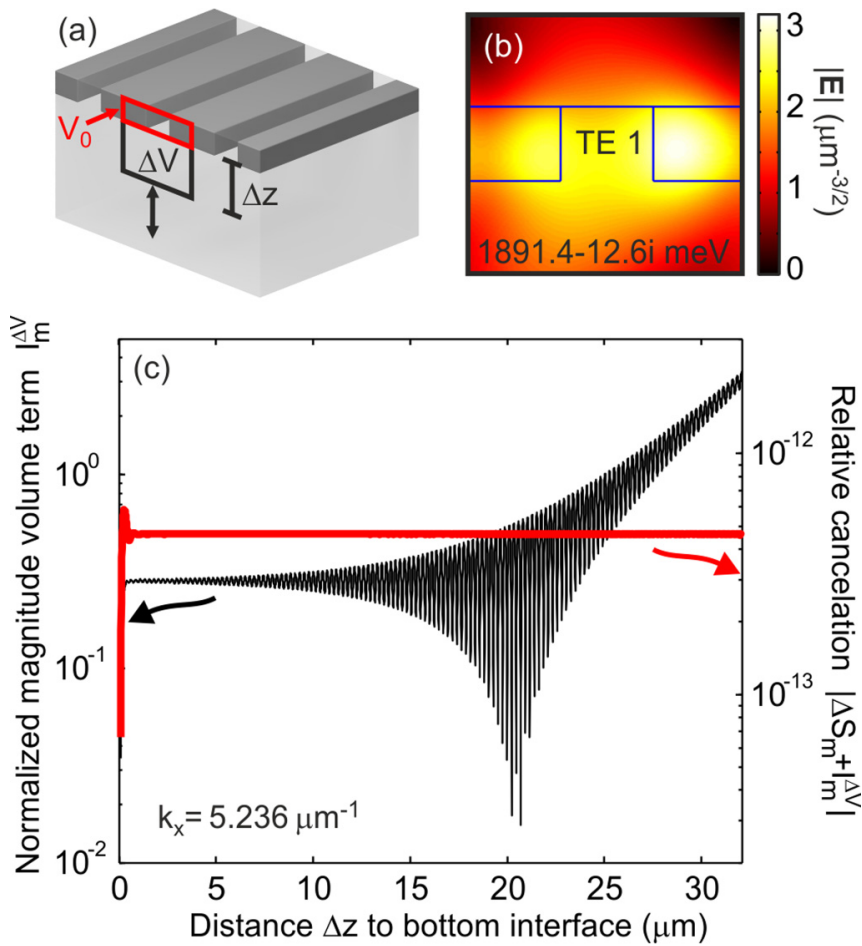

FIG. 2. The normalization of radiative resonances contains a volume and a surface contribution. The normalization volume spans in a lateral direction over one unit cell and contains at least the outermost interfaces to the homogenous top and bottom half spaces, as indicated by $\mathcal{V}_{0}$ in panel (a). Increasing the volume of integration for the normalization, e.g., by adding a volume of height $\Delta z$ in the substrate region, results in an additional volume contribution $I_{m}^{\Delta \mathcal{V}}$ that diverges for $\Delta z \rightarrow \infty$. Panel (b) displays the magnitude of the correctly normalized resonant electric field distribution in one unit cell for the same resonance as in Fig. 1(b). For this normalized field, the black solid line in panel (c) depicts $\left|I_{m}^{\Delta \mathcal{V}}\right|$. This divergence is compensated by the modification $\Delta S_{m}$ of the surface term, as indicated by the red solid line.

\section{RESONANT STATE EXPANSION}

For the sake of simplicity, we provide here the derivation of the resonant state expansion for nondispersive media, although the resonant state expansion is not limited to such systems [41]. Following the derivations in Ref. [40] and references therein, we can rewrite the Green's dyadic as (see Appendix D)

$$
\mathcal{G}\left(\mathbf{r}, \mathbf{r}^{\prime} ; \mathbf{k} ; \omega\right)=c^{2} \bigvee_{m} \frac{\mathbf{E}_{m}(\mathbf{r} ; \mathbf{k}) \otimes \mathbf{E}_{m}\left(\mathbf{r}^{\prime} ;-\mathbf{k}\right)}{2 \omega\left[\omega-\omega_{m}(\mathbf{k})\right]},
$$

where $\mathbf{E}_{m}$ and $\omega_{m}$ denote either the resonant field distribution and the frequency at a pole or their continuous values on a cut. Similarly, we define by the symbol $\$$ the combined contribution of a discrete sum over the resonant states and integrals along the cuts.

Next, we may consider the perturbations $\Delta \varepsilon(\mathbf{r})$ of our initial system. In the case of periodic structures, $\Delta \varepsilon$ is supposed to be periodic, and it must not exceed the region of the spatial inhomogeneities of the unperturbed system in the $z$ direction, where Eq. (21) is no longer applicable. Then, the modified eigenvalue equation (14) can be written in the following 
manner:

$$
\mathcal{L}\left(\omega_{\mu}\right) \mathbf{E}_{\mu}=-\frac{\omega_{\mu}^{2} \Delta \varepsilon(\mathbf{r})}{c^{2}} \mathbf{E}_{\mu} .
$$

Here, $\mathbf{E}_{\mu}$ denotes the resonant electric field distribution of the modified system with the eigenfrequency $\omega_{\mu}$. Using Eqs. (13) and (32), we can set up an integral equation for the new eigensolutions:

$$
\mathbf{E}_{\mu}(\mathbf{r} ; \mathbf{k})=-\sum_{m} \frac{\omega_{\mu} \mathbf{E}_{m}(\mathbf{r} ; \mathbf{k})}{2\left(\omega_{\mu}-\omega_{m}\right)} V_{m, \mu} .
$$

Here, we introduce the overlap integrals

$$
V_{m, \mu}=\int_{\mathcal{V}} d V \mathbf{E}_{m}(\mathbf{r} ;-\mathbf{k}) \cdot \Delta \varepsilon(\mathbf{r}) \mathbf{E}_{\mu}(\mathbf{r} ; \mathbf{k}) .
$$

Equation (34) motivates the ansatz

$$
\mathbf{E}_{\mu}(\mathbf{r} ; \mathbf{k})=\sum_{m} b_{m} \mathbf{E}_{m} .
$$

Substituting this ansatz into Eqs. (34) and (35) and comparing the coefficients for each resonant state $\mathbf{E}_{m}$ yields

$$
\omega_{m} b_{m}=\omega_{\mu} \sum_{m^{\prime}}\left(\hat{\delta}_{m, m^{\prime}}+\frac{1}{2} V_{m, m^{\prime}}\right) b_{m^{\prime}},
$$

where $\hat{\delta}_{m, m^{\prime}}$ denotes a Dirac $\delta$ for continuous contributions and a Kronecker $\delta$ for discrete states, and the matrix element $V_{m, m^{\prime}}$ contains the resonant electric field distributions of two unperturbed resonant states. Equation (37) is a generalized linear eigenvalue problem for eigenvalues $\omega_{\mu}$ and expansion coefficients $b_{m}$ that can be straightforwardly transformed into a standard linear eigenvalue equation [37]. Furthermore, in numerical implementations, the number of resonant states is limited to a finite number, and the cut contributions can be replaced by a discrete set of cut poles [38].

\section{A. First-order perturbation theory}

For small permittivity changes $\Delta \varepsilon$, it is often sufficient to take only one resonant state in Eq. (37) into account. This results in

$$
\Delta \omega \equiv \omega_{\mu}-\omega_{m} \approx-\frac{\omega_{m}}{2} V_{m, m} .
$$

If the permittivity change $\Delta \varepsilon$ is constant in a certain analyte volume $\mathcal{V}_{\mathrm{a}}$, the integral $V_{m, m}$ is proportional to $\Delta \varepsilon$. In this case, we can easily consider the limit $\Delta \varepsilon \rightarrow 0$. Using the refractive index instead of the permittivity, we then obtain the sensitivity of the eigenfrequency $\omega_{m}$ with respect to changes of the refractive index in $\mathcal{V}_{\mathrm{a}}$ for $\mathbf{k} \neq 0$ :

$$
\frac{\partial \omega_{m}}{\partial n}=-\omega_{m} n \int_{\mathcal{V}_{\mathrm{a}}} d V \mathbf{E}_{m}^{-} \cdot \mathbf{E}_{m}^{+} .
$$

Figure 3 depicts resonance energy (c) and linewidth (d) as a function of the refractive index in the high-index material of the periodic layer in the example geometry for two transverse magnetic resonances at $k_{x}=0.2 \mu \mathrm{m}^{-1}$. Black solid and blue dashed lines have been derived from Eq. (39) for a reference index of $n=2.5$ as $\omega_{\mu} \approx \omega_{m}+\Delta n \partial \omega_{m} / \partial n$, with black solid lines for the resonance at $2736.6-0.2 i \mathrm{meV}$ and blue dotted lines for that at $2737.5-5.2 i \mathrm{meV}$. The corresponding resonant electric field distributions are shown
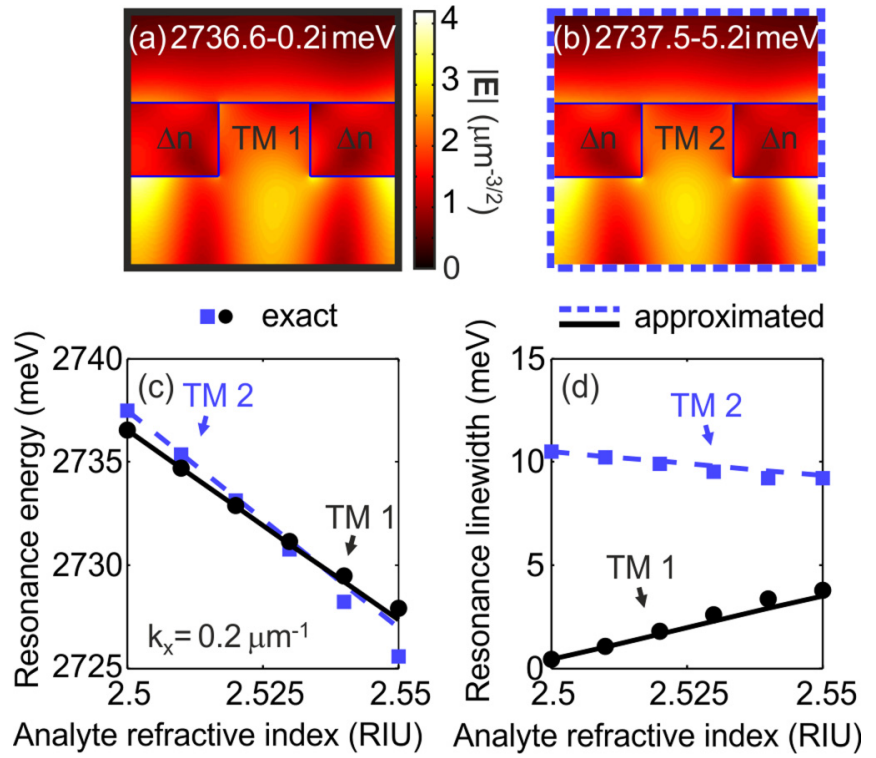

FIG. 3. Comparison of first-order perturbation theory (black solid and blue dashed lines) and numerically exact results (blue squares and black dots) for two transverse-magnetic (TM) resonances at $k_{x}=0.2 \mu \mathrm{m}^{-1}$. The resonance energies are 2736.6-0.2i meV and $2737.5-5.2 i \mathrm{meV}$; the corresponding normalized resonant electric field distributions can be seen in panels (a) and (b), respectively. Panels (c) and (d) show the resulting resonance energy and linewidth as a function of the refractive index in the high-index material of the periodic layer. The results of the first-order perturbation theory have been derived for a reference index of 2.5 .

in panels (a) and (b), respectively. Blue squares and black dots are the exact results of full numerical simulations of resonance energy and linewidth for the given refractive index values. For small changes of the refractive index, the exact results agree well with the first-order perturbation theory, thus justifying its applicability for predicting the dependence of the eigenfrequencies on the refractive index within the considered range of refractive index values.

Note that the electric field distributions of the two resonant states in Fig. 3 are rather similar. Both modes possess a nonzero resonance linewidth; i.e., they have a finite lifetime due to radiative losses. The main difference between these two modes is the behavior of the $(-1)$ diffraction order in the substrate. The resonant state in Fig. 3(a) exhibits exponential decay in this diffraction order, while the resonant state in Fig. 3(b) grows exponentially in the exterior, thus emitting light to the far field via this channel. Both modes grow exponentially in the zeroth order, i.e., radiate to the far field, and decay in all remaining orders. In Fig. 4, we show the dispersion of these two modes from the center of the first Brillouin zone to $k_{x}=0.21 \mu \mathrm{m}^{-1}$. The black dotted line in Fig. 4(a) denotes the position of the $(-1)$ Rayleigh anomaly, at which the $(-1)$ diffraction order changes its character on the real frequency axis from decaying to propagating when increasing $\omega$ or $k_{x}$. As we can see, the two resonant states from Fig. 3 can be traced continuously over the entire range.

In the first place, it might seem counterintuitive that there are regions in the first Brillouin zone in which both resonant states from Fig. 3 contribute simultaneously. In fact, the mode 

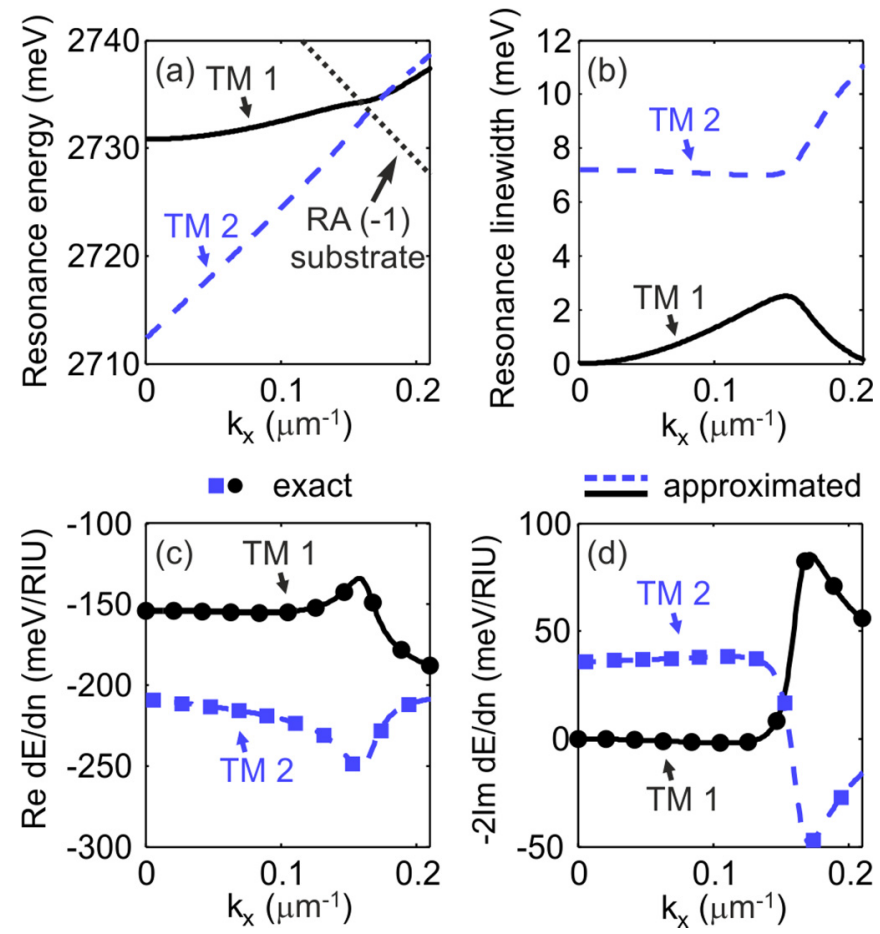

FIG. 4. Resonance energy (a) and linewidth (b) of the resonances depicted in Fig. 3 as a function of the in-plane momentum $k_{x}$. The black dotted line in panel (a) denotes the Rayleigh anomaly (RA) due to the opening of the $(-1)$ diffraction order in the substrate. As seen in panels (c) and (d), the predictions from the first-order perturbation theory (black solid and blue dashed lines) for the real and imaginary parts of $\partial E / \partial n(E=\hbar \omega)$ agree well with the numerically calculated derivatives $\Delta E / \Delta n$ (blue squares and black dots) for changes of the refractive index in the high-index material of the periodic layer.

labeled TM 1 (TM 2) determines the optical properties below (above) the opening of the $(-1)$ diffraction order on the real frequency axis. If the Rayleigh anomaly is far away from the real part of the resonance frequency of mode TM 1 (TM 2), the far-field spectra below (above) the Rayleigh anomaly exhibit the typical Lorentzian or Fano line shape of a single resonance. However, while the Rayleigh anomaly separates two regions on the real frequency axis, the behavior of the analytical continuation to complex frequencies is more sophisticated. As shown in Ref. [33], both resonant states do contribute equivalently to the shape of the far-field spectra if $k_{x}$ and $\omega$ are close to the Rayleigh anomaly and both eigenfrequencies are nearby. In our example geometry, this happens around $k_{x}=0.17 \mu \mathrm{m}^{-1}$ and $2730 \mathrm{meV}$ [see Fig. 4(a)]. In that region, the spectral behavior significantly deviates from the typical Lorentzian or Fano line shape (see Fig. 3 in Ref. [33]). Considering the functional dependence of the Green's dyadic in terms of complex $\kappa_{(-1)}^{\text {bot }}$ instead of $\omega$, it is even possible to remove the contribution of the cut $[33,39]$. Alternatively, the cut for the square root in Eq. (7) that starts at the $(-1)$ Rayleigh anomaly can be chosen such that both resonances are on the same Riemann sheet in the complex $\omega$ plane, so that both modes have to be taken into account in the expansion of the Green's dyadic, while the influence of the cut is reduced [55].
As Eq. (39) requires the correct normalization of the resonant electric field distributions, we can use the comparison of Eq. (39) with the numerical derivative $\Delta \omega / \Delta n$ for small $\Delta n$ in order to validate Eq. (30) at inclined incidence. Figure 4 shows the resulting real (c) and imaginary parts (d) of the slopes for the same modes as in panels (a) and (b). The perturbation theory (black solid and blue dashed lines) is in excellent agreement with the numerical derivative (blue squares and black dots).

It is worth mentioning that the sensitivity of the eigenfrequencies on the refractive index change in the high-index dielectric is rather large. The values of the figure of merit as $\left|\partial \omega_{m} / \partial n \operatorname{Im}\left(\omega_{m}\right)\right|$ exceed the values of the plasmonic antennas in Ref. [42]. This is because the quality factor of the modes is high and the refractive index change takes place in a region of high field intensities, thus rendering photonic crystal slabs as a good candidate for refractive index sensing if it is possible to realize the refractive index changes in the periodic layer.

\section{B. Two-mode resonant state expansion}

If there are resonant states with close eigenfrequencies on the complex frequency plane, or if the perturbation $\Delta \varepsilon$ becomes larger, the first-order perturbation theory is no longer accurate for describing the influence of the perturbation on the resonant states. Consequently, one has to use the resonant state expansion with several modes.

The simplest case is the two-mode resonant state expansion that is well suited to describe mode hybridization due to geometrical modifications. In this case, the resonant state expansion of Eq. (37) simplifies to the eigenvalue equation

$$
\omega_{\mu}\left(\begin{array}{l}
b_{1} \\
b_{2}
\end{array}\right)=\frac{1}{D}\left[\begin{array}{cc}
\omega_{1}\left(2+V_{22}\right) & -\omega_{2} V_{12} \\
-\omega_{1} V_{21} & \omega_{2}\left(2+V_{11}\right)
\end{array}\right]\left(\begin{array}{l}
b_{1} \\
b_{2}
\end{array}\right),
$$

with

$$
D=2+V_{11}+V_{22}+\frac{V_{11} V_{22}-V_{12} V_{21}}{2} .
$$

The analytical solutions of Eq. (40) are

$$
\omega_{\mu}^{ \pm}=\omega_{+} \pm \sqrt{\omega_{-}^{2}+\Delta}
$$

with

$$
\begin{gathered}
\omega_{ \pm}=\frac{\omega_{1}\left(2+V_{22}\right) \pm \omega_{2}\left(2+V_{11}\right)}{2 D}, \\
\Delta=\frac{\omega_{1} \omega_{2} V_{12} V_{21}}{D^{2}} .
\end{gathered}
$$

Notably, these solutions contain corrections to the unperturbed eigenfrequencies that do not vanish for $V_{12}=V_{21}=0$, providing the first-order result of Eq. (38) for weak perturbations. In standard coupled mode theory, e.g., the well-known plasmon hybridization [23], these contributions are not described.

Furthermore, the coupling terms in Eq. (40) are complex numbers with nonzero imaginary parts. Hence, the hybridized eigenfrequencies derived from Eq. (42) can exhibit a more diverse behavior than the simple hybridization in the weakand strong-coupling limits with avoided crossing in either the real or the imaginary part. To illustrate such behavior and to validate Eq. (42), we compare the results of Eq. (42) 
(a)
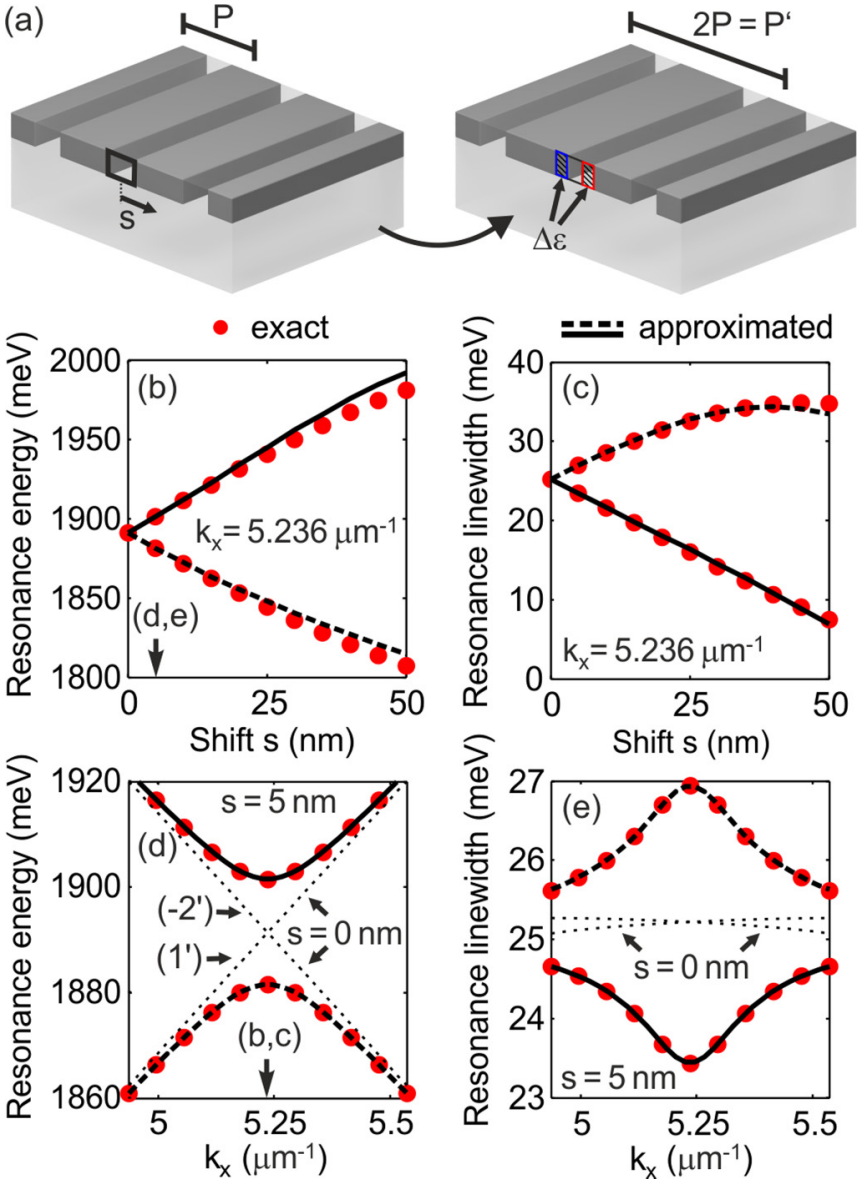

FIG. 5. (a) The geometry of Fig. 1(a) is modified by shifting the low-index region of the periodic layer in every second unit cell by a distance $s$. Thus, a superperiodicity is introduced, which allows coupling of the $\left(-2^{\prime}\right)$ and the $\left(1^{\prime}\right)$ fundamental transverse-electric resonances (thin black dotted lines) at the border of the first Brillouin zone of the superperiodic system at $k_{x}=\pi / 2 P \approx 5.236 \mu \mathrm{m}^{-1}$. Using the resonant state expansion (black solid and dashed lines) for these two resonances taken at $s=0$ allows for predicting the behavior of the resonance energies [panels (c) and (d)] and linewidths [panels (c) and (e)] of the coupled modes (red dots), shown here as a function of shift $s$ for $k_{x}=\pi / 2 P$ [panels (b) and (c)] and in-plane momentum $k_{x}$ for $s=5 \mathrm{~nm}$ [panels (d) and (e)].

with exact numerical solutions when introducing geometrical modifications in our example geometry. In particular, we modified every second unit cell by shifting the low-index region by a certain distance $s$ [see Fig. 5(a)]. Thus, we introduce a superlattice with period $P^{\prime}=2 P$. Consequently, the first Brillouin zone is downsized by a factor of 2 , and additional bands of quasiguided modes occur. For instance, there is a new mode of order $\left(1^{\prime}\right)$ that is created by diffracting the mode with order (1) by $2 \pi / P^{\prime}=\pi / P$, i.e., $\omega_{\left(1^{\prime}\right)}\left(k_{x}\right)=$ $\omega_{(1)}\left(k_{x}-\pi / P\right)$. At the border of the first Brillouin zone, this mode crosses the mode with order $\left(-2^{\prime}\right)$ when neglecting interaction between these modes. Equation (42) allows us to predict the mode hybridization due to the interaction.

Figure 5 shows a comparison between numerical calculations (red dots) and the results of Eq. (42) (black solid and dashed lines) for the transverse-electric mode at the crossing of the $\left(1^{\prime}\right)$ and $\left(-2^{\prime}\right)$ lines. Figures 5(b) and 5(c) depict the dependence of the resonance frequency and the linewidth, respectively, on the shift parameter $s$ at the border of the first Brillouin zone $\left(k_{x}=\pi / P^{\prime}\right)$. Even for shifts on the order of $50 \mathrm{~nm}$, the differences between exact results and the two-mode resonant state expansion are small. Figures 5(d) and 5(e) show the resonance frequency and the resonance linewidth, respectively, as a function of in-plane momentum $k_{x}$ for a constant shift of $s=5 \mathrm{~nm}$. The black dotted lines indicate the results in the uncoupled case with $s=0 \mathrm{~nm}$. It can be seen that Eq. (42) resembles well the behavior obtained by numerical simulations over the entire range. In particular, the two-mode resonant state expansion reproduces accurately the observed avoided crossing for both the resonance frequency and the resonance linewidth.

\section{SUMMARY}

We have derived an analytical mode normalization for oneand two-dimensional planar periodic structures. Our results have been validated by comparing full numerical simulations with the resonant state expansion for one and two modes. Our work will be relevant for designing advanced geometries for refractive index sensing and for obtaining a deeper understanding of the influence of geometrical perturbations on the optical properties of micro- and nanostructures including arrays of plasmonic nanoantennas as well as photonic crystal slabs. Due to the high sensitivity of the resonance frequencies and the high quality factor of the modes in the investigated photonic crystal slab, such structures seem to be promising candidates for refractive index sensing devices [56]. In addition, the correct normalization is necessary for calculating the Purcell factor, for estimating the changes of the spontaneous emission rate of quantum emitters located inside the micro- and nanostructures [10].

\section{ACKNOWLEDGMENTS}

We acknowledge support from ERC Advanced Grant COMPLEXPLAS as well as DFG SPP 1839, BMBF, MWK Baden-Württemberg, the VW Foundation, and the BadenWürttemberg Stiftung. E.A.M. and W.L. acknowledge support from the Sêr Cymru National Research Network. N.A.G, S.G.T., and E.A.M. acknowledge support from the Russian Foundation of Basic Research (Grant No. RFBR 16-2903283). S.G.T. thanks the DFG for a Mercator Fellowship in the framework of the SPP 1839.

\section{APPENDIX A: RELATIONS IN HOMOGENEOUS EXTERIOR}

The following relations are valid in the exterior homogeneous layers. Consider $\psi_{-\mathbf{K}}(\mathbf{r} ; \omega)$ and $\psi_{\mathbf{K}^{\prime}}(\mathbf{r} ; \omega)$ being solutions of the scalar wave equation. Here, we take $-\mathbf{K}=$ $-\mathbf{k}-\mathbf{G}$ and $\mathbf{K}^{\prime}=\mathbf{k}+\mathbf{G}^{\prime}$, i.e., having opposite in-plane momentum $\mathbf{k}$, which corresponds to the usual field conjugation in our theory [see, e.g., Eqs. (23) and (27)]. In this case,

$$
\begin{aligned}
0 & =\psi_{-\mathbf{K}}\left(\nabla^{2}+\frac{\omega^{2} \varepsilon}{c^{2}}\right) \psi_{\mathbf{K}^{\prime}}-\psi_{\mathbf{K}^{\prime}}\left(\nabla^{2}+\frac{\omega^{2} \varepsilon}{c^{2}}\right) \psi_{-\mathbf{K}} \\
& =\psi_{-\mathbf{K}} \nabla^{2} \psi_{\mathbf{K}^{\prime}}-\psi_{\mathbf{K}^{\prime}} \nabla^{2} \psi_{-\mathbf{K}} .
\end{aligned}
$$


Integrating over an arbitrary volume and using Green's second identity results in

$$
\begin{aligned}
0 & =\int_{\mathcal{V}} d V\left(\psi_{-\mathbf{K}} \nabla^{2} \psi_{\mathbf{K}^{\prime}}-\psi_{\mathbf{K}^{\prime}} \nabla^{2} \psi_{-\mathbf{K}}\right) \\
& =\oint_{\partial \mathcal{V}} d S\left(\psi_{-\mathbf{K}} \partial_{s} \psi_{\mathbf{K}^{\prime}}-\psi_{\mathbf{K}^{\prime}} \partial_{s} \psi_{-\mathbf{K}}\right) .
\end{aligned}
$$

If we chose a volume that spans in lateral directions over one unit cell, the surface integrals at the boundary of the unit cell vanish due to the periodicity of $\psi_{-\mathbf{K}} \partial_{s} \psi_{\mathbf{K}^{\prime}}$ and $\psi_{\mathbf{K}^{\prime}} \partial_{s} \psi_{-\mathbf{K}}$. Furthermore, if we select the top or bottom surface to be a plane normal to the $z$ direction, we can derive from the orthogonality relation (10) that

$$
\begin{aligned}
& \int_{\mathcal{S}_{\mathbf{u}}} d x d y\left(\psi_{-\mathbf{K}} \frac{\partial \psi_{\mathbf{K}^{\prime}}}{\partial \Delta z}-\psi_{\mathbf{K}^{\prime}} \frac{\partial \psi_{-\mathbf{K}}}{\partial \Delta z}\right) \\
& =i\left(\kappa_{\mathbf{K}^{\prime}}-\kappa_{\mathbf{K}}\right) \delta_{\mathbf{K}, \mathbf{K}^{\prime}}=0 .
\end{aligned}
$$

Thus, without any restrictions on the remaining top or bottom surface, we obtain from Eqs. (A2) and (A3) that any surface integral over one unit cell must vanish:

$$
\int_{\mathcal{S}_{\mathbf{u}}} d S\left(\psi_{-\mathbf{K}} \partial_{s} \psi_{\mathbf{K}^{\prime}}-\psi_{\mathbf{K}^{\prime}} \partial_{s} \psi_{-\mathbf{K}}\right)=0
$$

As the polarization vectors $\hat{\mathbf{E}}_{\mathbf{K}}^{p, \alpha}(\omega)$ are independent of $\mathbf{r}$, Eq. (A4) also holds for solutions of the vector wave equation:

$$
\int_{\mathcal{S}_{\mathrm{u}}} d S\left(\mathbf{E}_{-\mathbf{K}}^{p, \alpha} \cdot \partial_{s} \mathbf{E}_{\mathbf{K}^{\prime}}^{p^{\prime}, \alpha}-\mathbf{E}_{\mathbf{K}^{\prime}}^{p^{\prime}, \alpha} \cdot \partial_{s} \mathbf{E}_{-\mathbf{K}}^{p, \alpha}\right)=0 .
$$

\section{APPENDIX B: INTEGRAL RELATIONS FOR NORMALIZATION}

This section is devoted to deriving Eqs. (25) to (27) as well as Eq. (31) from Eq. (23). Equation (23) can be written as

$$
1+\delta_{\omega_{m}, 0}=\lim _{\omega \rightarrow \omega_{m}}\left(I_{1}+I_{2}\right)
$$

with

$$
I_{1} \equiv \int_{\mathcal{V}} d V \frac{\mathbf{E}_{m}^{-} \cdot \omega^{2} \varepsilon(\omega) \tilde{\mathbf{E}}_{m}^{+}(\omega)-\tilde{\mathbf{E}}_{m}^{+}(\omega) \cdot \omega_{m}^{2} \varepsilon\left(\omega_{m}\right) \mathbf{E}_{m}^{-}}{\omega^{2}-\omega_{m}^{2}},
$$

which immediately provides Eq. (26) in the limit $\omega \rightarrow \omega_{m}$, and

$$
I_{2} \equiv c^{2} \int_{\mathcal{V}} d V \frac{\tilde{\mathbf{E}}_{m}^{+}(\omega) \cdot \nabla \times \nabla \times \mathbf{E}_{m}^{-}-\mathbf{E}_{m}^{-} \cdot \nabla \times \nabla \times \tilde{\mathbf{E}}_{m}^{+}(\omega)}{\omega^{2}-\omega_{m}^{2}} .
$$

The following vector relation [40] can be used in order to transform the volume integral in Eq. (B3) into a surface integral:

$$
\begin{aligned}
\mathbf{A} & =\nabla \times \nabla \times \mathbf{B}-\mathbf{B} \cdot \nabla \times \nabla \times \mathbf{A} \\
& =\nabla \cdot\left[\mathbf{A}(\boldsymbol{\nabla} \cdot \mathbf{B})-\mathbf{B}(\boldsymbol{\nabla} \cdot \mathbf{A})+\sum_{j=x, y, z} B_{j} \nabla A_{j}-A_{j} \nabla B_{j}\right] .
\end{aligned}
$$

When being in the homogeneous exterior, we can use that $\nabla \cdot \mathbf{E}_{m}\left(\mathbf{r}_{>}^{\alpha} ; \mathbf{k}\right)=0$ and $\nabla \cdot \tilde{\mathbf{E}}_{m}\left(\mathbf{r}_{>}^{\alpha} ; \mathbf{k} ; \omega\right)=0$, which yields

$$
I_{2}=\frac{S_{m}^{\partial \mathcal{V}}(\omega)}{\omega^{2}-\omega_{m}^{2}}
$$

The term $S_{m}^{\partial \mathcal{V}}(\omega)$ is defined in Eq. (27) and contains an integration over the surface $\partial \mathcal{V}$ of the normalization volume $\mathcal{V}$. As the surface contributions at the periodic boundaries vanish, we only have to consider the top and bottom surfaces, for which we deduce from the plane wave expansion (28) and Eq. (A5) that $S_{m}^{\partial \mathcal{V}}\left(\omega_{m}\right)=0$. Hence, adding this zero to Eq. (B5), we obtain that $I_{2}$ has the form of a difference quotient, which results in the $\omega^{2}$ derivative at $\omega_{m}$ when taking the limit $\omega \rightarrow \omega_{m}$ :

$$
\lim _{\omega \rightarrow \omega_{m}} I_{2}=\lim _{\omega \rightarrow \omega_{m}} \frac{S_{m}^{\partial \mathcal{V}}(\omega)-S_{m}^{\partial \mathcal{V}}\left(\omega_{m}\right)}{\omega^{2}-\omega_{m}^{2}}=\left.\frac{\partial S_{m}^{\partial \mathcal{V}}}{\partial\left(\omega^{2}\right)}\right|_{\omega_{m}} .
$$

To calculate the derivative in Eq. (B6), i.e., the surface contribution in Eq. (25), we may now use the plane wave expansions of $\mathbf{E}_{m}$ and $\tilde{\mathbf{E}}_{m}$. Equations (28) and (29) then provide

$$
\left.\frac{\partial S_{m}^{\partial \mathcal{V}}}{\partial\left(\omega^{2}\right)}\right|_{\omega_{m}}=\left.c^{2} \sum_{p, \alpha} \sum_{\mathbf{K}, \mathbf{K}^{\prime}} a_{-\mathbf{K}}^{p, \alpha} \frac{\partial c_{\mathbf{K}, \mathbf{K}^{\prime}}^{p, \alpha} S_{\mathbf{K}, \mathbf{K}^{\prime}}^{\alpha}}{\partial\left(\omega^{2}\right)}\right|_{\omega_{m}},
$$

with $\alpha=\{$ top, bot $\}, p=\{$ TE,TM $\}$,

$$
c_{\mathbf{K}, \mathbf{K}^{\prime}}^{p, \alpha}(\omega)=\tilde{a}_{\mathbf{K}^{\prime}}^{p, \alpha}(\omega) \hat{\mathbf{E}}_{-\mathbf{K}}^{p, \alpha}\left(\omega_{m}\right) \cdot \hat{\mathbf{E}}_{\mathbf{K}^{\prime}}^{p, \alpha}(\omega),
$$

and

$$
S_{\mathbf{K}, \mathbf{K}^{\prime}}^{\alpha}(\omega)=\int_{\mathcal{S}_{\mathbf{u}}} d S\left[\psi_{-\mathbf{K}}^{\alpha}\left(\omega_{m}\right) \partial_{s} \psi_{\mathbf{K}^{\prime}}^{\alpha}(\omega)-\psi_{\mathbf{K}^{\prime}}^{\alpha}(\omega) \partial_{s} \psi_{-\mathbf{K}}^{\alpha}\left(\omega_{m}\right)\right] .
$$

Note that $a_{-\mathbf{K}}^{p, \alpha}$ originates from the plane wave expansion of $\mathbf{E}_{m}$, so that it does not depend on $\omega$. In contrast, $c_{\mathbf{K}, \mathbf{K}^{\prime}}^{p, \alpha}(\omega) \propto \tilde{a}_{\mathbf{K}^{\prime}}^{p, \alpha}(\omega)$, so that it is in general frequency dependent. Therefore, the derivative on the right-hand side of Eq. (B7) can be expressed as

$$
\begin{aligned}
\left.\frac{\partial c_{\mathbf{K}, \mathbf{K}^{\prime}}^{p, \alpha} S_{\mathbf{K}, \mathbf{K}^{\prime}}^{\alpha}}{\partial\left(\omega^{2}\right)}\right|_{\omega_{m}}= & \left.S_{\mathbf{K}, \mathbf{K}^{\prime}}^{\alpha}\left(\omega_{m}\right) \frac{\partial c_{\mathbf{K}, \mathbf{K}^{\prime}}^{p, \alpha}}{\partial\left(\omega^{2}\right)}\right|_{\omega_{m}} \\
& +\left.c_{\mathbf{K}, \mathbf{K}^{\prime}}^{p, \alpha}\left(\omega_{m}\right) \frac{\partial S_{\mathbf{K}, \mathbf{K}^{\prime}}^{\alpha}}{\partial\left(\omega^{2}\right)}\right|_{\omega_{m}} .
\end{aligned}
$$

Owing to Eq. (A4), $S_{\mathbf{K}, \mathbf{K}^{\prime}}^{\alpha}\left(\omega_{m}\right)=0$, so that we obtain

$$
\left.\frac{\partial S_{m}^{\partial \mathcal{V}}}{\partial\left(\omega^{2}\right)}\right|_{\omega_{m}}=\left.c^{2} \sum_{p, \alpha} \sum_{\mathbf{K}, \mathbf{K}^{\prime}} a_{-\mathbf{K}}^{p, \alpha} c_{\mathbf{K}, \mathbf{K}^{\prime}}^{p, \alpha}\left(\omega_{m}\right) \frac{\partial S_{\mathbf{K}, \mathbf{K}^{\prime}}^{\alpha}}{\partial\left(\omega^{2}\right)}\right|_{\omega_{m}},
$$

with

$$
\begin{aligned}
\left.\frac{\partial S_{\mathbf{K}, \mathbf{K}^{\prime}}^{\alpha}}{\partial\left(\omega^{2}\right)}\right|_{\omega_{m}}= & \left.i \frac{\partial \kappa_{\mathbf{K}^{\prime}}^{\alpha}}{\partial\left(\omega^{2}\right)}\right|_{\omega_{m}} \int_{\mathcal{S}_{\mathbf{u}}} d S\left[\psi_{-\mathbf{K}}^{\alpha}\left(\omega_{m}\right) \partial_{s} \Delta z^{\alpha} \psi_{\mathbf{K}^{\prime}}^{\alpha}\left(\omega_{m}\right)\right. \\
& \left.-\Delta z^{\alpha} \psi_{\mathbf{K}^{\prime}}^{\alpha}\left(\omega_{m}\right) \partial_{s} \psi_{-\mathbf{K}}^{\alpha}\left(\omega_{m}\right)\right] .
\end{aligned}
$$

In summary, the surface contribution to Eq. (25) can be calculated from Eq. (B11), which only depends on the expansion coefficients $a_{ \pm \mathbf{K}}^{p, \alpha}$ of the resonant field distributions. Hence, the normalization in Eq. (25) does not depend on the spatial profile of the underlying source term $\sigma_{m}$, because both the volume and the surface term do depend only on $\lim _{\omega \rightarrow \omega_{m}} \tilde{\mathbf{E}}_{m}(\omega)=\mathbf{E}_{m}$; 
i.e., they are independent of the actual frequency dependence of $\tilde{\mathbf{E}}_{m}$, determined by the source term $\sigma_{m}$, and can be directly calculated from the resonant field distributions. Furthermore, the surface contribution can be derived for arbitrary surfaces. Using Eq. (7) and choosing $\mathcal{S}_{\mathrm{u}}$ to be a plane normal to the $z$ axis straightforwardly results in Eq. (31).

\section{APPENDIX C: GREEN'S DYADIC AND RECIPROCITY}

Consider two currents, $\mathbf{j}_{1}^{+}(\mathbf{r}) \equiv \mathbf{j}_{1}(\mathbf{r} ; \mathbf{k} ; \omega) \quad$ and $\mathbf{j}_{2}^{-}(\mathbf{r}) \equiv \mathbf{j}_{2}(\mathbf{r} ;-\mathbf{k} ; \omega)$, that are the sources of the fields $\mathbf{E}_{1}^{+}$and $\mathbf{E}_{2}^{-}$. Taking the wave equation (1) for $\mathbf{j}_{1}^{+}$, multiplying it with $\mathbf{E}_{2}^{-}$, and subtracting the wave equation for $\mathbf{j}_{2}^{-}$multiplied with $\mathbf{E}_{1}^{+}$provides

$$
\begin{aligned}
& \frac{4 \pi i \omega}{c^{2}}\left(\mathbf{E}_{2}^{-} \cdot \mathbf{j}_{1}^{+}-\mathbf{E}_{1}^{+} \cdot \mathbf{j}_{2}^{-}\right) \\
& \quad=\mathbf{E}_{2}^{-} \cdot\left(\nabla \times \nabla \times \mathbf{E}_{1}^{+}\right)-\mathbf{E}_{1}^{+} \cdot\left(\nabla \times \nabla \times \mathbf{E}_{2}^{-}\right) .
\end{aligned}
$$

When integrating this equation over a volume $\mathcal{V}$ that extends to the exterior homogeneous regions, we can use the vector relations of Eq. (B4) to transform the right-hand side into a surface integral:

$$
\begin{aligned}
& \frac{4 \pi i \omega}{c^{2}} \int_{\mathcal{V}} d V\left(\mathbf{E}_{2}^{-} \cdot \mathbf{j}_{1}^{+}-\mathbf{E}_{1}^{+} \cdot \mathbf{j}_{2}^{-}\right) \\
& =\oint_{\partial \mathcal{V}} d S\left(\mathbf{E}_{1}^{+} \cdot \partial_{s} \mathbf{E}_{2}^{-}-\mathbf{E}_{2}^{-} \cdot \partial_{s} \mathbf{E}_{1}^{+}\right) .
\end{aligned}
$$

If the volume of integration spans over one unit cell in the lateral direction, the right-hand side of this equation equals zero due to the periodicity of the fields and Eq. (A5).

We can now replace the fields in Eq. (C2) using the Green's dyadic:

$$
\begin{aligned}
0= & \iint_{\mathcal{V}} d V d V^{\prime}\left[\mathbf{j}_{1}^{+}(\mathbf{r}) \cdot \mathcal{G}\left(\mathbf{r}, \mathbf{r}^{\prime} ;-\mathbf{k} ; \omega\right) \mathbf{j}_{2}^{-}\left(\mathbf{r}^{\prime}\right)\right. \\
& \left.-\mathbf{j}_{2}^{-}(\mathbf{r}) \cdot \mathcal{G}\left(\mathbf{r}, \mathbf{r}^{\prime} ; \mathbf{k} ; \omega\right) \mathbf{j}_{1}^{+}\left(\mathbf{r}^{\prime}\right)\right] .
\end{aligned}
$$

Because this relation has to be fulfilled for arbitrary $\mathbf{j}_{1}^{+}$and $\mathbf{j}_{2}^{-}$, it immediately follows that

$$
\mathcal{G}\left(\mathbf{r}, \mathbf{r}^{\prime} ; \mathbf{k} ; \omega\right)=\left[\mathcal{G}\left(\mathbf{r}^{\prime}, \mathbf{r} ;-\mathbf{k} ; \omega\right)\right]^{\mathrm{T}},
$$

where the superscript denotes the matrix transpose.

Let us now derive the representation of the Green's dyadic in terms of resonant states. Formally, the cut contributions in Eq. (17) can be considered as a series of integrals, each denoting a continuous amount of poles on the cut. Therefore, we may define the tensor $\mathcal{R}_{m}\left(\mathbf{r}, \mathbf{r}^{\prime} ; \mathbf{k}\right)$ such that

$$
\mathcal{G}\left(\mathbf{r}, \mathbf{r}^{\prime} ; \mathbf{k} ; \omega\right)=\sum_{m} \frac{\mathcal{R}_{m}\left(\mathbf{r}, \mathbf{r}^{\prime} ; \mathbf{k}\right)}{\omega-\omega_{m}(\mathbf{k})} .
$$

Accordingly, Eq. (16) becomes

$$
\begin{aligned}
\mathbf{E}_{m}(\mathbf{r} ; \mathbf{k})= & \lim _{\omega \rightarrow \omega_{m}} \sum_{m^{\prime}} \frac{1}{c^{2}} \frac{\omega^{2}-\omega_{m}^{2}}{\omega-\omega_{m^{\prime}}} \\
& \times \int_{\mathcal{V}} d V^{\prime} \mathcal{R}_{m^{\prime}}\left(\mathbf{r}, \mathbf{r}^{\prime} ; \mathbf{k}\right) \cdot \sigma_{m}\left(\mathbf{r}^{\prime} ; \mathbf{k}\right) .
\end{aligned}
$$

This relation must be fulfilled independently of the exact form of $\sigma_{m}$, which requires that $\mathcal{R}_{m}$ has the form of an outer vector product [38], i.e., $\mathcal{R}_{m}\left(\mathbf{r}, \mathbf{r}^{\prime} ; \mathbf{k}\right) \propto \mathbf{E}_{m}(\mathbf{r} ; \mathbf{k}) \otimes \mathbf{F}_{m}\left(\mathbf{r}^{\prime} ; \mathbf{k}\right)$, with fields $\mathbf{F}_{m}\left(\mathbf{r}^{\prime} ; \mathbf{k}\right)$ that have to be determined from the properties of the Green's dyadic. If we consider reciprocal systems, the reciprocity principle requires that $\mathcal{R}_{m}\left(\mathbf{r}, \mathbf{r}^{\prime} ; \mathbf{k}\right)=$ $\left[\mathcal{R}_{m}\left(\mathbf{r}^{\prime}, \mathbf{r} ;-\mathbf{k}\right)\right]^{\mathrm{T}}$, so that $\mathbf{F}_{m}\left(\mathbf{r}^{\prime} ; \mathbf{k}\right)=\mathbf{E}_{m}\left(\mathbf{r}^{\prime} ;-\mathbf{k}\right)$. Therefore, we can deduce the ansatz $\mathcal{R}_{m}\left(\mathbf{r}, \mathbf{r}^{\prime} ; \mathbf{k}\right)=c^{2} \mathbf{E}_{m}(\mathbf{r} ; \mathbf{k}) \otimes$ $\mathbf{E}_{m}\left(\mathbf{r}^{\prime} ;-\mathbf{k}\right) / 2 \omega_{m}$, which results in Eq. (12). Similar considerations for the cut contributions lead to Eq. (22).

\section{APPENDIX D: SUM RULE AND CLOSURE RELATION}

In this section, we follow the derivations in Ref. [10] for obtaining the sum rule and closure relations for the resonant states in periodic structures with nondispersive permittivity functions. Substituting $\mathcal{G}$ from Eqs. (21) and (22) into Eq. (12) and using that $\mathbf{E}_{m}$ and $\mathbf{E}_{\omega_{\mathbf{K}}}$ obey Eq. (14), we obtain

$$
\frac{\varepsilon(\mathbf{r})}{2} \sum_{m} \frac{\omega^{2}-\omega_{m}^{2}}{\omega_{m}\left(\omega-\omega_{m}\right)} \mathbf{E}_{m}^{+} \otimes \mathbf{E}_{m}^{-}=\sum_{\mathbf{R}} \mathbb{1} \delta\left(\mathbf{r}-\mathbf{r}^{\prime}-\mathbf{R}\right) .
$$

The second fraction on the left-hand side of Eq. (D1) reduces to $1+\omega / \omega_{m}$, and since the right-hand side does not depend on the frequency $\omega$, this splits into the closure relation

$$
\frac{\varepsilon(\mathbf{r})}{2} \sum_{m} \mathbf{E}_{m}^{+} \otimes \mathbf{E}_{m}^{-}=\sum_{\mathbf{R}} \mathbb{1} \delta\left(\mathbf{r}-\mathbf{r}^{\prime}-\mathbf{R}\right)
$$

and the sum rule

$$
\sum_{m} \frac{\mathbf{E}_{m}^{+} \otimes \mathbf{E}_{m}^{-}}{\omega_{m}}=0
$$

Using this sum rule, we can rewrite the Green's dyadic of Eq. (21) in the compact form of Eq. (32).
[1] F. Aieta, M. A. Kats, P. Genevet, and F. Capasso, Science 347, 1342 (2015).

[2] X. Yin, T. Steinle, L. Huang, T. Taubner, M. Wuttig, T. Zentgraf, and H. Giessen, Light: Sci. Appl. (to be published, 2017).

[3] G. Dolling, M. Wegener, C. M. Soukoulis, and S. Linden, Opt. Express 15, 11536 (2007).

[4] J. Valentine, S. Zhang, T. Zentgraf, E. Ulin-Avila, D. A. Genov, G. Bartal, and X. Zhang, Nature (London) 455, 376 (2008).

[5] N. Liu, M. Mesch, T. Weiss, M. Hentschel, and H. Giessen, Nano Lett. 10, 2342 (2010).
[6] G. M. Akselrod, H. Huang, T. B. Hoang, P. T. Bowen, L. Su, D. R. Smith, and M. H. Mikkelsen, Adv. Mater. 27, 8028 (2015).

[7] A. Blanco, E. Chomski, S. Grabtchak, M. Ibisate, S. John, S. W. Leonard, C. Lopez, F. Meseguer, H. Miguez, J. P. Mondia, G. A. Ozin, O. Toader, and H. M. van Driel, Nature (London) 405, 437 (2000).

[8] P. St. J. Russell, Science 299, 358 (2003).

[9] C. Sauvan, J. P. Hugonin, I. S. Maksymov, and P. Lalanne, Phys. Rev. Lett. 110, 237401 (2013). 
[10] E. A. Muljarov and W. Langbein, Phys. Rev. B 94, 235438 (2016).

[11] E. M. Purcell, Phys. Rev. 69, 681 (1946).

[12] G. Zheng, H. Mühlenbernd, M. Kenney, G. Li, T. Zentgraf, and S. Zhang, Nat. Nanotechnol. 10, 308 (2015).

[13] A. O. Govorov, Z. Fan, P. Hernandez, J. M. Slocik, and R. R. Naik, Nano Lett. 10, 374 (2010).

[14] M. L. Nesterov, X. Yin, M. Schäferling, H. Giessen, and T. Weiss, ACS Photon. 4, 578 (2016).

[15] V. I. Belotelov, L. L. Doskolovich, and A. K. Zvezdin, Phys. Rev. Lett. 98, 077401 (2007).

[16] D. Floess, T. Weiss, S. G. Tikhodeev, and H. Giessen, Phys. Rev. Lett. 117, 063901 (2016).

[17] S. Arnold, M. Khoshsima, I. Teraoka, S. Holler, and F. Vollmer, Opt. Lett. 28, 272 (2003).

[18] A. Unger and M. Kreiter, J. Phys. Chem. C 113, 12243 (2009).

[19] G. Mie, Ann. Phys. 25, 377 (1908).

[20] W. A. Murray and W. L. Barnes, Adv. Mater. 19, 3771 (2007).

[21] T. Fujita, Y. Sato, T. Kuitani, and T. Ishihara, Phys. Rev. B 57, 12428 (1998).

[22] A. Christ, S. G. Tikhodeev, N. A. Gippius, J. Kuhl, and H. Giessen, Phys. Rev. Lett. 91, 183901 (2003).

[23] P. Nordlander, C. Oubre, E. Prodan, K. Li, and M. I. Stockman, Nano Lett. 4, 899 (2004).

[24] N. A. Gippius, T. Weiss, S. G. Tikhodeev, and H. Giessen, Opt. Express 18, 7569 (2010).

[25] B. Gallinet and O. J. F. Martin, Opt. Express 19, 22167 (2011).

[26] R. W. Wood, Philos. Mag. 4, 396 (1902).

[27] V. N. Astratov, D. M. Whittaker, I. S. Culshaw, R. M. Stevenson, M. S. Skolnick, T. F. Krauss, and R. M. De La Rue, Phys. Rev. B 60, R16255(R) (1999).

[28] S. G. Tikhodeev, A. L. Yablonskii, E. A. Muljarov, N. A. Gippius, and T. Ishihara, Phys. Rev. B 66, 045102 (2002).

[29] D. N. Chigrin, A. V. Lavrinenko, and C. M. S. Torres, Opt. Express 12, 617 (2004).

[30] B. Auguié and W. L. Barnes, Phys. Rev. Lett. 101, 143902 (2008).

[31] R. Singh, C. Rockstuhl, and W. Zhang, Appl. Phys. Lett. 97, 241108 (2010).

[32] N. Feth, M. König, M. Husnik, K. Stannigel, J. Niegemann, K. Busch, M. Wegener, and S. Linden, Opt. Express 18, 46545 (2010).

[33] A. B. Akimov, N. A. Gippius, and S. G. Tikhodeev, JETP Lett. 93, 427 (2011).

[34] R. Sammut and A. W. Snyder, Appl. Opt. 15, 1040 (1976).
[35] H. M. Lai, P. T. Leung, K. Young, P. W. Barber, and S. C. Hill, Phys. Rev. A 41, 5187 (1990).

[36] L. A. Weinstein, Open Resonators and Open Waveguides (Golem, Boulder, CO, 1969).

[37] E. A. Muljarov, W. Langbein, and R. Zimmermann, Europhys. Lett. 92, 50010 (2010).

[38] M. B. Doost, W. Langbein, and E. A. Muljarov, Phys. Rev. A 87, 043827 (2013).

[39] L. J. Armitage, M. B. Doost, W. Langbein, and E. A. Muljarov, Phys. Rev. A 89, 053832 (2014).

[40] M. B. Doost, W. Langbein, and E. A. Muljarov, Phys. Rev. A 90, 013834 (2014).

[41] E. A. Muljarov and W. Langbein, Phys. Rev. B 93, 075417 (2016).

[42] T. Weiss, M. Mesch, M. Schäferling, H. Giessen, W. Langbein, and E. A. Muljarov, Phys. Rev. Lett. 116, 237401 (2016).

[43] Q. Bai, M. Perrin, C. Sauvan, J. P. Hugonin, and P. Lalanne, Opt. Express 21, 27371 (2013).

[44] P. T. Kristensen, C. V. Vlack, and S. Hughes, Opt. Lett. 37, 1649 (2012).

[45] P. T. Kristensen, R.-C. Ge, and S. Hughes, Phys. Rev. A 92, 053810 (2015).

[46] E. A. Muljarov and W. Langbein, Phys. Rev. A 96, 017801 (2017).

[47] T. Weiss, G. Granet, N. A. Gippius, S. G. Tikhodeev, and H. Giessen, Opt. Express 17, 8051 (2009).

[48] T. Weiss, N. A. Gippius, S. G. Tikhodeev, G. Granet, and H. Giessen, J. Opt. Soc. Am. A 28, 238 (2011).

[49] D. A. Bykov and L. L. Doskolovich, J. Lightwave Technol. 31, 793 (2013).

[50] J. Yang, H. Giessen, and P. Lalanne, Nano Lett. 15, 3439 (2015).

[51] T. Zentgraf, A. Christ, J. Kuhl, N. A. Gippius, S. G. Tikhodeev, D. Nau, and H. Giessen, Phys. Rev. B 73, 115103 (2006).

[52] D. Nau, A. Schönhardt, C. Bauer, A. Christ, T. Zentgraf, J. Kuhl, M. W. Klein, and H. Giessen, Phys. Rev. Lett. 98, 133902 (2007).

[53] D. D. Solnyshkov, T. Weiss, G. Malpuech, and N. A. Gippius, Appl. Phys. Lett. 99, 111110 (2011).

[54] G. B. Arfken and H. J. Weber, Mathematical Methods for Physicists, 6th ed. (Elsevier, London, 2011).

[55] S. V. Lobanov, G. Zoriniants, W. Langbein, and E. A. Muljarov, Phys. Rev. A 95, 053848 (2017).

[56] K. Awazu, C. Rockstuhl, M. Fujimaki, N. Fukuda, J. Tominaga, T. Komatsubara, T. Ikeda, and Y. Ohki, Opt. Express 15, 2592 (2007). 